Article

\title{
A Credit Rating Model in a Fuzzy Inference System Environment
}

\author{
Amir Karbassi Yazdi ${ }^{1}$, Thomas Hanne ${ }^{2, * \mathbb{C}}$, Yong J. Wang ${ }^{3}$ and Hui-Ming Wee ${ }^{4}(\mathbb{D}$ \\ 1 Young Researchers and Elite Club, South Tehran Branch, Islamic Azad University, North Iranshahr 233, \\ Tehran 19585/466, Iran \\ 2 Institute for Information Systems, University of Applied Sciences and Arts Northwestern Switzerland, \\ Riggenbachstrasse 16, 4600 Olten, Switzerland \\ 3 Department of Marketing, West Chester University, 700 South High Street, West Chester, PA 19383, USA \\ 4 Industrial \& Systems Engineering Department, Chung Yuan Christian University, Taoyuan, \\ Taoyuan City 32023, Taiwan \\ * Correspondence: thomas.hanne@fhnw.ch
}

Received: 21 May 2019; Accepted: 30 June 2019; Published: 9 July 2019

check for

\begin{abstract}
One of the most important functions of an export credit agency (ECA) is to act as an intermediary between national governments and exporters. These organizations provide financing to reduce the political and commercial risks in international trade. The agents assess the buyers based on financial and non-financial indicators to determine whether it is advisable to grant them credit. Because many of these indicators are qualitative and inherently linguistically ambiguous, the agents must make decisions in uncertain environments. Therefore, to make the most accurate decision possible, they often utilize fuzzy inference systems. The purpose of this research was to design a credit rating model in an uncertain environment using the fuzzy inference system (FIS). In this research, we used suitable variables of agency ratings from previous studies and then screened them via the Delphi method. Finally, we created a credit rating model using these variables and FIS including related IF-THEN rules which can be applied in a practical setting.
\end{abstract}

Keywords: credit rating; export credit agencies; uncertainty environment; fuzzy inference system; Delphi method

\section{Introduction}

Due to the strong expansion of international trade, many countries have established export credit agencies (ECAs) to protect exporters from bankruptcy due to political and commercial risks. Their agents evaluate foreign buyers and determine whether to grant credit to these exporters to protect them from risks. The agents evaluate the buyers based on their countries' sovereign credit. In this paper, we use a Fuzzy Inference System (FIS) to evaluate exporters' credit in an uncertain environment.

For each contract, the sellers need to know about the financial situation of the buyers. This study helps them to evaluate the ability of buyers to repay their debt, and to determine the probability of default. There are two types of credit ratings: (1) sovereign credit rating and (2) corporate credit rating. To ascertain the risk level of the buyers' investment, we evaluate them as well as their government. Credit risk can be analyzed using various financial tools, which are affected by political, social, and economic factors.

Decision-making is an essential function of any enterprise. It is very difficult for decision makers (DMs) to utilize quantitative variables since many evaluation attributes are vague. For this type of uncertain environment, linguistic and verbal scales can be helpful in making an appropriate decision. Thus, the fuzzy set theory of linguistic variables can express the preferences of decision makers in an uncertain environment. 
Fuzzy logic and fuzzy set theory, introduced independently by Zadeh [1] and Klaua, have inspired many scholars over the decades. Since then, fuzzy models have found wide areas of application, including various economically important areas. For instance, in [2], an adaptive neuro-fuzzy inference system was used for selecting vehicle routes under uncertainty conditions. In [3], a similar type of model was used for determining economic order quantities (such as for procurement or production planning). Based on the Boston Consulting Group (BCG) portfolio matrix, a neuro-fuzzy approach is elaborated in [4] for analyzing human resources. Further application examples include the multiobjective route planning for the transport of hazardous material [5] or location planning for city logistics [6].

In addition, many studies have been published on using fuzzy modelling in the context of buyer evaluation and credit scoring, including those by Akkoç [7] who investigated loan defaults. Due to financial crises, many financial institutes aim for accurate credit scoring models. Ramkumar and Busi [8] utilized a modified analytic network process (ANP) and fuzzy inference system to establish a risk assessment model for third-party e-procurement systems. As part of a modified ANP, decision makers are encouraged to express their preferences verbally rather than via a numerical rating system. Yazdi et al. [9] used an adaptive neural fuzzy inference system to create inputs, outputs, membership functions, and fuzzy rules. The results indicated that these sets of constraints lead to similar constraint categories with output fuzzy trained systems. Moghadam et al. [10] used the FIS method to map the model. They found that a fuzzy inference system could be helpful in this type of research. The results showed that two factors were particularly effective for mapping via FIS. In the first step, FIS was used to weight factors. In the next step, the factors were integrated using FIS, and the final step revealed the results of exploratory boreholes. Dash and Dash [11] tested a model to predict stock prices by using the Self-Evolving Recurrent Neuro-Fuzzy Inference System (SERNFIS) and modified differential harmony search. They used stock market time series data utilizing diverse time frames. The Takagi-Sugeno-Kang (TSK) model and fuzzy IF-THEN rules were also used.

In order to evaluate buyers who represent companies or individuals, these agencies must consider some of the following indicators or use models created by credit rating agencies such as Moody's, S\&P, Fitch, Capital Intelligence, Euler Hermes, Japan Credit Rating Agency, etc. [12]. For example, the Export Guarantee Fund of Iran (EGFI) is the only credit rating agency that uses a customized model to evaluate the credit ratings of buyers' companies to determine whether to grant credit to exporters (EGFI website). Considering that this agency uses both quantitative and qualitative indicators for evaluating these companies, it is of utmost importance to create the most accurate model possible. Furthermore, because the economic situation of Iran is uncertain, using a model for translating ambiguous and qualitative indicators is crucial to obtaining the most accurate assessment [13]. A fuzzy inference system is a robust computerized technique for decision-making in such an environment.

For this study, a three-stage hybrid adaptive neuro-fuzzy inference system for credit scoring was used as a statistical technique. This model was tested in Turkey's national banks using a 10-fold cross process [7]. The results revealed that this model performed better than linear discriminate analysis, logistic regression analysis, and an artificial neural network. The contributions this study makes are the use of the fuzzy inference system to evaluate credit ratings in uncertain environments. Moreover, our methodology includes building the proposed model by finding a similar one, which most accurately represents the challenging economic situation in Iran. This model is customized via the Delphi method using experts' opinions [7].

In this paper, we present the application of fuzzy modeling methods to a problem of rating companies with respect to credit decisions. Based on the input from experts of a rating agency, ranking criteria are determined and assessed in terms of linguistic variables. Subsequently, fuzzy rules for an FIS are determined. The approach is applied under practical conditions by an ECA in order to cope better with difficult economic conditions and severe budget limitations. In particular, decisions are based on a transparent model instead of ad hoc assumptions and decisions which affect the quality of decisions. 
This paper is organized as follows: Section 2 is the literature review. Section 3 presents the research methodology. Section 4 describes the data analysis. Finally, Section 5 presents the conclusions and formulates suggestions for future research.

\section{Literature Review and Basic Definitions}

\subsection{Literature Review}

Since the establishment of ECAs, bankruptcy due to political and commercial risks has decreased dramatically. While the ECAs are designed to protect exporters, they are also beneficial to global trade and succeed in encouraging companies to establish more credit. However, because the worldwide economic situation is unstable, making a truly accurate decision is very difficult. To accomplish this, many scholars have studied the credit ratings of companies in uncertain environments. For instance, Al-Najjar and Al-Najjar [14] showed how to measure corporate credit ratings in emerging markets. They used a neural network and a clustering method to rate major companies in Jordan during 2000 to 2007. Bian [15] looked at how the Chinese credit rating agencies were developed. He argued that Chinese companies should have a customized model for evaluating various companies and that they must focus on transparency. Chen and Cheng [16] established a hybrid model for credit rating by employing the rough set theory in an uncertain environment using factor analysis. Then, they used a learning algorithm for establishing decision-making rules. The result showed that this hybrid model was more effective than previous models. Doumpos et al. [17] used a multiple attribute decision-making (MADM) method based on linear programming and structural data to rate European firms using accounting data. Gibilaro and Mattarocci [18] investigated how rating agencies can grant credit based on customers' portfolios. They analyzed 20,389 companies using the S\&P, Moody's, and Fitch agencies. They evaluated these companies via the Herfindahl-Hirschman index and customer lifetime value. Gogas et al. [19] showed how to calculate the credit rating of banks. They evaluated 94 American banks by logic probability regression. The results showed that only $84 \%$ of those bank ratings were accurate. Orsenigo and Vercellis [20] used linear and nonlinear techniques to determine credit ratings for banks. They used double-bounded tree-connected Isomaps and principal component analysis to assess European, American, and Asian banks; they then classified the banks based on financial and non-financial indicators. Ozturk et al. [21] applied artificial intelligence techniques, such as classification and regression trees, multilayer perception, and support vector machines to measure sovereign credit ratings. Pasricha et al. [22] used Markov regenerative processes to establish a credit rating model. They applied the technique to find matrices of migration probability. They showed how past and current data influenced the ratings. $\mathrm{Hu}$ and $\mathrm{Hu}$ [23] studied the effect of sovereign ratings on bank stock returns in the European Union. They found that positive sovereign ratings did not lead to a bank's stock price reaction; however, negative events caused negative sovereign rating events.

\subsection{Fuzzy Inference System}

One of the advantages of fuzzy sets is their ability to translate qualitative and vague information into deterministic and quantitative data. This method has been applied in many different industries worldwide in spite of some conflicting opinions about its methodology [1]. The most common application of this method is decision-making, especially in an uncertain environment. To implement this method, we introduce the definition and notation of sets below. The first definition related to the membership function is as follows:

Definition 1. Fuzzy membership: $\mu_{\mathrm{A}}$ is defined as a membership function or characteristic function with values $\mu_{\mathrm{A}}\left((\mathrm{x}) \in[0 ; 1]\right.$ for $\mathrm{x} \in \mathrm{X}$. If $\mathrm{A} \subseteq \mathrm{X}$ indicates a crisp (traditional) set, then $\mu_{\mathrm{A}}$ assigns a value 0 or 1 to each member of $X . \mu_{A}(x)=1$ if $x \in A$; this means that $x$ has full membership. $\mu_{A}(X)=0$ if $x \notin A$; this means that $X$ does not have any membership in $X[1]$. 
The membership function of $\widetilde{A}$ can be specified, for instance, as a triangular, a trapezoidal, a Gaussian function, or a sigmoid function. Moreover, logical operations can be used including AND, OR, and NOT [24].

Definition 2. Triangular fuzzy numbers: $\widetilde{A}=\left\{x, \mu_{\tilde{A}} \mid x \in X\right\}$. There are three parameters of a triangular fuzzy membership function, $a, m$, and $b$. The corresponding function is defined in Equation (1) [1]:

$$
\mu_{\widetilde{A}}(x)= \begin{cases}\frac{x-a}{m-a}, & a \leq x \leq m \\ \frac{b-x}{b-m}, & m \leq x \leq b \\ 0, & \text { otherwise }\end{cases}
$$

The third definition relates to the product of fuzzy numbers based on a t-norm operator.

Definition 3. The product of fuzzy numbers: The fuzzy numbers of $\widetilde{A}$ and $\widetilde{B}$ are produced by $t$-norm operators, as shown in Equation (2) [1]:

$$
\mu_{\widetilde{\mathrm{A}}}(\mathrm{x}) \operatorname{AND} \mu_{\widetilde{\mathrm{B}}}(\mathrm{y})=-\mu_{\widetilde{\mathrm{A}}}(\mathrm{x}) \times \mu_{\widetilde{\mathrm{B}}}(\mathrm{y})
$$

Based on this method, one of the most important applications is the fuzzy inference system (FIS), which uses IF-THEN rules based on fuzzy membership functions. In FIS, all inputs based on a membership function change to an output membership function according to IF-THEN rules. There are various systems that translate the inputs to output membership functions in the FIS. However, we discuss only the two most essential [25-27]. Mamdani's output membership function is based on defuzzification. After computation with fuzzy numbers, these numbers must be transferred into crisp numbers to make decisions easier. There are many methods available for this. In our study, we use the mean method for changing fuzzy numbers to crisp numbers.

The FIS consists of four steps. First, the inputs and their degree of fuzziness are defined. Second, we set up some fuzzy operators. Third, we determine the weights of each IF-THEN rule and use them to obtain the decision. Fourth, all rules are entered either as inputs or operators.

\section{Research Methodology}

Because research on export credit agencies is rather novel, there is plenty of scope for study. In this study, we attempt to introduce a new method for credit rating agencies based on the Moody method. It is customized for the Export Guarantee Fund of Iran (EGFI). The research questions are as follows:

1. Which variables are suitable for the EGFI as well as for other credit rating agencies?

2. How does the uncertain environment affect these variables?

In order to determine the variables for determining credit ratings, we introduced Moody's model and experts' opinions on these variables. These variables are interest coverage ratio, current ratio, quick ratio, ownership structure, country risk and so on. After extracting these variables, we evaluated them using the Delphi method as follows:

(a) These variables were sent to the experts of the EGFI to determine which ones were suitable for credit rating agencies.

(b) Within the Delphi method, a 5-point Likert scale was used.

(c) When the average of the experts' opinions was less than 4, this variable was eliminated.

Tables 1 and 2 show the computation of variables and their extractions. The results show that, among 23 variables, only 19 should be used for ranking companies. 
Table 1. Preferences of experts (decision makers (DM)) regarding input variables. DEBT-TO-EQUITY RATIO (Earnings Before Interest, Taxes, Depreciation and Amortization(EBITDA)), Debt-Service Coverage Ratio (DSCR), Return On Equity (ROE), Definitions of these input variables are provided in Table 3.

\begin{tabular}{cccccccccc}
\hline VARIABLES & DM1 & DM2 & DM3 & DM4 & DM5 & DM6 & DM7 & DM8 & DM9 \\
\hline DEBT-TO-EQUITY RATIO & 4 & 5 & 4 & 5 & 3 & 4 & 5 & 5 & 4 \\
DEBT RATIO TO EBITDA & 5 & 5 & 5 & 4 & 3 & 4 & 5 & 5 & 4 \\
DSCR & 5 & 4 & 4 & 5 & 5 & 5 & 5 & 4 & 3 \\
INTEREST COVERAGE RATIO & 3 & 4 & 5 & 5 & 5 & 4 & 5 & 4 & 5 \\
CASH FROM OPERATING ACTIVITIES & 5 & 5 & 5 & 5 & 4 & 5 & 3 & 5 & 4 \\
RATIO TO TOTAL SALES & 4 & 4 & 5 & 4 & 5 & 4 & 5 & 4 & 3 \\
ROE & 5 & 5 & 5 & 4 & 5 & 4 & 3 & 4 & 5 \\
OPERATING PROFIT MARGIN & 4 & 5 & 4 & 5 & 4 & 5 & 4 & 3 & 4 \\
CURRENT RATIO & 5 & 5 & 5 & 5 & 4 & 5 & 4 & 5 & 3 \\
QUICK RATIO & 4 & 4 & 4 & 5 & 4 & 5 & 4 & 5 & 3 \\
ASSET TURNOVER & 4 & 4 & 4 & 5 & 5 & 4 & 5 & 3 & 5 \\
MANAGEMENT STRUCTURE & 4 & 3 & 4 & 5 & 4 & 3 & 3 & 2 & 4 \\
SUCCESSION PLANNING & 3 & 3 & 3 & 3 & 4 & 5 & 3 & 4 & 2 \\
STRATEGIC PLANNING & 4 & 4 & 4 & 5 & 5 & 5 & 3 & 4 & 5 \\
CORPORATE GOVERNANCE & 3 & 5 & 4 & 4 & 5 & 4 & 5 & 4 & 3 \\
OWNERSHIP STRUCTURE & 4 & 4 & 5 & 4 & 5 & 4 & 3 & 3 & 5 \\
DIVERSIFICATION OF INCOME & 5 & 5 & 4 & 5 & 4 & 5 & 4 & 3 & 4 \\
PAYMENT RECORDS & 3 & 3 & 3 & 2 & 3 & 4 & 5 & 3 & 4 \\
COMPANY AUDITORS & 3 & 4 & 4 & 5 & 5 & 5 & 5 & 3 & 4 \\
QUALITY AND TRANSPARENCY OF & 4 & 5 & 5 & 5 & 4 & 5 & 3 & 4 & 5 \\
REPORTING & 3 & 4 & 5 & 5 & 5 & 5 & 4 & 5 & 4 \\
COMPETITIVENESS & 3 & 3 & 3 & 4 & 3 & 4 & 2 & 3 & 4 \\
POSITION IN THE INDUSTRY/MARKET & 4 & 5 & 5 & 5 & 5 & 4 & 4 & 3 & 4 \\
RISK OF INDUSTRY & & & & & & & &
\end{tabular}

Table 2. Results of the Delphi method.

\begin{tabular}{|c|c|c|c|}
\hline & VARIABLES & AVERAGE SCORE & ACCEPT/REJECT \\
\hline 1 & debt-to-equity ratio & 4.333333333 & Accept \\
\hline 2 & debt ratio to EBITDA & 4.444444444 & Accept \\
\hline 3 & DSCR & 4.444444444 & Accept \\
\hline 4 & interest coverage ratio & 4.444444444 & Accept \\
\hline 5 & cash from operating activities ratio to total sales & 4.555555556 & Accept \\
\hline 6 & ROE & 4.222222222 & Accept \\
\hline 7 & operating profit margin & 4.444444444 & Accept \\
\hline 8 & current ratio & 4.222222222 & Accept \\
\hline 9 & quick ratio & 4.555555556 & Accept \\
\hline 10 & asset turnover & 4.222222222 & Accept \\
\hline 11 & management structure & 4.333333333 & Accept \\
\hline 12 & succession planning & 3.555555556 & Reject \\
\hline 13 & strategic planning & 3.333333333 & Reject \\
\hline 14 & corporate governance & 4.333333333 & Accept \\
\hline 15 & ownership structure & 4.111111111 & Accept \\
\hline 16 & diversification of income & 4.111111111 & Accept \\
\hline 17 & payment records & 4.333333333 & Accept \\
\hline 18 & company auditors & 3.333333333 & Reject \\
\hline 19 & quality and transparency of reporting & 4.222222222 & Accept \\
\hline 20 & competitiveness & 4.444444444 & Accept \\
\hline 21 & position in the industry/market & 4.444444444 & Accept \\
\hline 22 & risk of industry & 3.222222222 & Reject \\
\hline 23 & groups of country risk & 4.333333333 & Accept \\
\hline
\end{tabular}


Table 3. References of variables.

\begin{tabular}{cc}
\hline Factor & References \\
\hline debt-to-equity ratio & {$[28,29]$} \\
debt ratio to EBITDA & {$[30,31]$} \\
DSCR & {$[32,33]$} \\
interest coverage ratio & {$[34,35]$} \\
cash from operating activities ratio to total sales & {$[36]$} \\
ROE & {$[37-39]$} \\
operating profit margin & {$[40,41]$} \\
current ratio & {$[42,43]$} \\
quick ratio & {$[41,44,45]$} \\
asset turnover & {$[46,47]$} \\
management structure & {$[48]$} \\
corporate governance & {$[49,50]$} \\
ownership structure & {$[50,51]$} \\
diversification of income & {$[52,53]$} \\
payment records & {$[54]$} \\
quality and transparency of reporting & {$[55,56]$} \\
competitiveness & {$[57-59]$} \\
company position & {$[60]$} \\
country risk & {$[61,62]$} \\
\hline
\end{tabular}

We used MATLAB (version 2015b, created by Cleve Moler, University of New Mexico, matrix laboratory, USA) and a fuzzy inference system to evaluate the variables.

\section{Data Analysis}

As shown in the previous section, the input variables (accepted variables according to Table 2) were debt-to-equity ratio, debt ratio to EBITDA (earnings before interest, tax, depreciation and amortization), DSCR (debt service coverage ratio), interest coverage ratio, cash from operating activities ratio to total sales, ROE (return on equity), operating profit margin, current ratio, quick ratio, asset turnover, management structure, corporate governance, ownership structure, diversification of income, payment records, quality and transparency of reporting, competitiveness, company position, and country risk. Table 3 provides an overview of these variables together with further references.

Table 4 shows the ranges of ratings for these variables, which were based on the opinions of experts from the Delphi method. These ranges are based on the broad experiences of the experts and provide valuable information to specify the FIS.

Table 4. Ranges of each variable and relationships to linguistic variables.

\begin{tabular}{|c|c|c|}
\hline Variable & & \\
\hline \multirow{6}{*}{ debt-to-equity ratio } & $x>150 \%$ & very poor \\
\hline & $125 \% \leq x \leq 150 \%$ & almost very poor \\
\hline & $100 \% \leq x \leq 125 \%$ & poor \\
\hline & $75 \% \leq x \leq 100 \%$ & average \\
\hline & $50 \% \leq x \leq 75 \%$ & good \\
\hline & $x<50 \%$ & very good \\
\hline
\end{tabular}


Table 4. Cont.

\begin{tabular}{|c|c|c|}
\hline Variable & \multicolumn{2}{|c|}{ Range } \\
\hline \multirow{5}{*}{ debt ratio to EBITDA } & $x>5$ & very poor \\
\hline & $4 \leq x \leq 5$ & poor \\
\hline & $3 \leq x \leq 4$ & average \\
\hline & $2 \leq x \leq 3$ & good \\
\hline & $x<2$ & very good \\
\hline \multirow{5}{*}{ DSCR } & $x<1$ & very poor \\
\hline & $1 \leq x \leq 1.25$ & poor \\
\hline & $1.25 \leq x \leq 1.75$ & average \\
\hline & $1.75 \leq x \leq 2.5$ & good \\
\hline & $x>2.5$ & very good \\
\hline \multirow{5}{*}{ interest coverage ratio } & $x<1$ & very poor \\
\hline & $1 \leq x \leq 2$ & poor \\
\hline & $2 \leq x \leq 4$ & average \\
\hline & $4 \leq x \leq 7$ & good \\
\hline & $x>7$ & very good \\
\hline \multirow{5}{*}{ cash from operating activities ratio to total sales } & $x<5 \%$ & very poor \\
\hline & $5 \% \leq x \leq 12.5 \%$ & poor \\
\hline & $12.5 \% \leq x \leq 20 \%$ & average \\
\hline & $20 \% \leq x \leq 30 \%$ & good \\
\hline & $x>30 \%$ & very good \\
\hline \multirow{5}{*}{ ROE } & $x<5 \%$ & very poor \\
\hline & $5 \% \leq x \leq 10 \%$ & poor \\
\hline & $10 \% \leq x \leq 15 \%$ & average \\
\hline & $15 \% \leq x \leq 20 \%$ & good \\
\hline & $x>20 \%$ & very good \\
\hline \multirow{5}{*}{ operating profit margin } & $x<5 \%$ & very poor \\
\hline & $5 \% \leq x \leq 10 \%$ & poor \\
\hline & $10 \% \leq x \leq 17.5 \%$ & average \\
\hline & $17.5 \% \leq x \leq 25 \%$ & good \\
\hline & $x>25 \%$ & very good \\
\hline \multirow{5}{*}{ current ratio } & $x<1$ & very poor \\
\hline & $1 \leq x \leq 1.25$ & poor \\
\hline & $1.25 \leq x \leq 1.75$ & average \\
\hline & $1.75 \leq x \leq 2.5$ & good \\
\hline & $x>2.5$ & very good \\
\hline
\end{tabular}


Table 4. Cont.

\begin{tabular}{|c|c|c|}
\hline Variable & \multicolumn{2}{|c|}{ Range } \\
\hline \multirow{5}{*}{ quick ratio } & $x<0.5$ & very poor \\
\hline & $0.5 \leq x \leq 0.75$ & poor \\
\hline & $0.75 \leq x \leq 1.25$ & average \\
\hline & $1.25 \leq x \leq 1.75$ & good \\
\hline & $x>1.75$ & very good \\
\hline \multirow{5}{*}{ asset turnover } & $x<0.5$ & very poor \\
\hline & $0.5 \leq x \leq 1$ & poor \\
\hline & $1 \leq x \leq 1.5$ & average \\
\hline & $1.5 \leq x \leq 2$ & good \\
\hline & $x>2$ & very good \\
\hline \multirow{5}{*}{ management structure } & \multicolumn{2}{|c|}{ inadequate } \\
\hline & \multicolumn{2}{|c|}{ below average } \\
\hline & \multicolumn{2}{|c|}{ average } \\
\hline & \multicolumn{2}{|c|}{ above average } \\
\hline & \multicolumn{2}{|c|}{ adequate } \\
\hline \multirow{5}{*}{ corporate governance } & \multicolumn{2}{|c|}{ weakness } \\
\hline & \multicolumn{2}{|c|}{ average } \\
\hline & \multicolumn{2}{|c|}{ satisfied } \\
\hline & \multicolumn{2}{|c|}{ very good } \\
\hline & \multicolumn{2}{|c|}{ excellent } \\
\hline & \multicolumn{2}{|c|}{ weakness } \\
\hline & \multicolumn{2}{|c|}{ average } \\
\hline ownership structure & \multicolumn{2}{|c|}{ satisfied } \\
\hline & \multicolumn{2}{|c|}{ very good } \\
\hline & \multicolumn{2}{|c|}{ excellent } \\
\hline \multirow{5}{*}{ diversification of income } & \multicolumn{2}{|c|}{ one specific income } \\
\hline & \multicolumn{2}{|c|}{ limited } \\
\hline & \multicolumn{2}{|c|}{ balanced } \\
\hline & \multicolumn{2}{|c|}{ highly diversified income } \\
\hline & \multicolumn{2}{|c|}{ very highly diversified income } \\
\hline \multicolumn{3}{|c|}{ very poor } \\
\hline \multirow{4}{*}{ payment records } & \multicolumn{2}{|c|}{ poor } \\
\hline & \multicolumn{2}{|c|}{ average } \\
\hline & & \\
\hline & & \\
\hline
\end{tabular}


Table 4. Cont.

\begin{tabular}{|c|c|}
\hline Variable & Range \\
\hline \multirow{5}{*}{ quality and transparency of reporting } & very poor \\
\hline & poor \\
\hline & average \\
\hline & good \\
\hline & very good \\
\hline \multirow{5}{*}{ competitiveness } & enemy \\
\hline & aggressive \\
\hline & average \\
\hline & suitable \\
\hline & without threat \\
\hline \multirow{5}{*}{ company position } & starter \\
\hline & small performer \\
\hline & middle performer \\
\hline & main performer \\
\hline & market leader \\
\hline \multirow{7}{*}{ country risk } & highest risk \\
\hline & almost high risk \\
\hline & often risk \\
\hline & middle risk \\
\hline & low risk \\
\hline & very low risk \\
\hline & no risk \\
\hline
\end{tabular}

Table 5 shows how we created the membership function of each variable and IF-THEN rules. Then, we employed the IF-THEN rules to categorize agencies based on the input variables.

We utilized numerous IF-THEN rules to evaluate the input variables as shown in Table 5 . These rules are based on input variables and their membership functions. We extracted the data of each company by considering the practical variables we obtained via the Delphi method. We then identified the highest percentage, average, and the lowest percentage of the triangular fuzzy membership function of each variable. As specified above (Equation (1)), a triangular membership function uses the parameters $a, b$, and $m$. The percentage values are denoted as alpha-cuts and are calculated according to [63]. An alpha-cut corresponds to the set of elements whose membership grades are greater than or equal to the specified value of alpha. Equation (3) shows how the alpha-cut is calculated:

$$
[\mathrm{A}]^{\alpha}=[a-m(1-\alpha), a+b(1-\alpha)] .
$$

We combined them based on the FIS and separated them into seven categories based on their levels of risk. This study helps managers make decisions and decreases the probability of a company defaulting. 
Table 5. IF-THEN rules. For each of the seven evaluation categories, a respective rule is shown.

\begin{tabular}{|c|c|c|c|c|c|c|c|c|c|c|c|c|c|c|c|c|c|c|c|}
\hline If & If & If & If & If & If & If & If & If & If & If & If & If & If & If & If & If & If & If & then \\
\hline $\begin{array}{l}\text { very } \\
\text { poor }\end{array}$ & $\begin{array}{l}\text { very } \\
\text { poor }\end{array}$ & $\begin{array}{l}\text { very } \\
\text { poor }\end{array}$ & $\begin{array}{l}\text { very } \\
\text { poor }\end{array}$ & $\begin{array}{l}\text { very } \\
\text { poor }\end{array}$ & $\begin{array}{l}\text { very } \\
\text { poor }\end{array}$ & $\begin{array}{l}\text { very } \\
\text { poor }\end{array}$ & $\begin{array}{l}\text { very } \\
\text { poor }\end{array}$ & $\begin{array}{l}\text { very } \\
\text { poor }\end{array}$ & $\begin{array}{l}\text { very } \\
\text { poor }\end{array}$ & inadequate & weakness & weakness & $\begin{array}{l}\text { one } \\
\text { specific } \\
\text { income }\end{array}$ & $\begin{array}{l}\text { very } \\
\text { poor }\end{array}$ & $\begin{array}{l}\text { very } \\
\text { poor }\end{array}$ & enemy & starter & $\begin{array}{l}\text { highest } \\
\text { risk }\end{array}$ & 7 \\
\hline $\begin{array}{l}\text { very } \\
\text { poor }\end{array}$ & $\begin{array}{l}\text { very } \\
\text { poor }\end{array}$ & $\begin{array}{l}\text { very } \\
\text { poor }\end{array}$ & $\begin{array}{l}\text { very } \\
\text { poor }\end{array}$ & $\begin{array}{l}\text { very } \\
\text { poor }\end{array}$ & $\begin{array}{l}\text { very } \\
\text { poor }\end{array}$ & $\begin{array}{l}\text { very } \\
\text { poor }\end{array}$ & $\begin{array}{l}\text { very } \\
\text { poor }\end{array}$ & $\begin{array}{l}\text { very } \\
\text { poor }\end{array}$ & $\begin{array}{l}\text { very } \\
\text { poor }\end{array}$ & Inadequate & weakness & weakness & $\begin{array}{l}\text { one } \\
\text { specific } \\
\text { income }\end{array}$ & $\begin{array}{l}\text { very } \\
\text { poor }\end{array}$ & $\begin{array}{l}\text { very } \\
\text { poor }\end{array}$ & enemy & starter & $\begin{array}{l}\text { almost } \\
\text { high } \\
\text { risk }\end{array}$ & 6 \\
\hline $\begin{array}{l}\text { almost } \\
\text { poor }\end{array}$ & poor & poor & poor & poor & poor & poor & poor & poor & poor & $\begin{array}{c}\text { below } \\
\text { average }\end{array}$ & average & average & limited & poor & poor & aggressive & $\begin{array}{c}\text { small } \\
\text { performer }\end{array}$ & $\begin{array}{c}\text { often } \\
\text { risk }\end{array}$ & 5 \\
\hline poor & average & average & average & average & average & average & average & average & average & average & satisfied & satisfied & balanced & average & average & average & $\begin{array}{c}\text { middle } \\
\text { performer }\end{array}$ & $\begin{array}{l}\text { middle } \\
\text { risk }\end{array}$ & 4 \\
\hline average & good & good & good & good & good & good & good & good & good & $\begin{array}{c}\text { above } \\
\text { average }\end{array}$ & $\begin{array}{l}\text { very } \\
\text { good }\end{array}$ & $\begin{array}{l}\text { very } \\
\text { good }\end{array}$ & $\begin{array}{c}\text { highly } \\
\text { diversified } \\
\text { income }\end{array}$ & good & good & suitable & $\begin{array}{c}\text { main } \\
\text { performer }\end{array}$ & $\begin{array}{l}\text { low } \\
\text { risk }\end{array}$ & 3 \\
\hline good & $\begin{array}{l}\text { very } \\
\text { good }\end{array}$ & $\begin{array}{l}\text { very } \\
\text { good }\end{array}$ & $\begin{array}{l}\text { very } \\
\text { good }\end{array}$ & $\begin{array}{l}\text { very } \\
\text { good }\end{array}$ & $\begin{array}{l}\text { very } \\
\text { good }\end{array}$ & $\begin{array}{l}\text { very } \\
\text { good }\end{array}$ & $\begin{array}{l}\text { very } \\
\text { good }\end{array}$ & $\begin{array}{l}\text { very } \\
\text { good }\end{array}$ & $\begin{array}{l}\text { very } \\
\text { good }\end{array}$ & adequate & excellent & excellent & $\begin{array}{c}\text { very } \\
\text { highly } \\
\text { diversified } \\
\text { income }\end{array}$ & $\begin{array}{l}\text { very } \\
\text { good }\end{array}$ & $\begin{array}{l}\text { very } \\
\text { good }\end{array}$ & $\begin{array}{l}\text { without } \\
\text { threat }\end{array}$ & $\begin{array}{l}\text { market } \\
\text { leader }\end{array}$ & $\begin{array}{l}\text { very } \\
\text { low } \\
\text { risk }\end{array}$ & 2 \\
\hline $\begin{array}{l}\text { very } \\
\text { good }\end{array}$ & $\begin{array}{l}\text { very } \\
\text { good }\end{array}$ & $\begin{array}{l}\text { very } \\
\text { good }\end{array}$ & $\begin{array}{l}\text { very } \\
\text { good }\end{array}$ & $\begin{array}{l}\text { very } \\
\text { good }\end{array}$ & $\begin{array}{l}\text { very } \\
\text { good }\end{array}$ & $\begin{array}{l}\text { very } \\
\text { good }\end{array}$ & $\begin{array}{l}\text { very } \\
\text { good }\end{array}$ & $\begin{array}{l}\text { very } \\
\text { good }\end{array}$ & $\begin{array}{l}\text { very } \\
\text { good }\end{array}$ & adequate & excellent & excellent & $\begin{array}{c}\text { very } \\
\text { highly } \\
\text { diversified } \\
\text { income }\end{array}$ & $\begin{array}{l}\text { very } \\
\text { good }\end{array}$ & $\begin{array}{l}\text { very } \\
\text { good }\end{array}$ & $\begin{array}{l}\text { without } \\
\text { threat }\end{array}$ & $\begin{array}{l}\text { market } \\
\text { leader }\end{array}$ & no risk & 1 \\
\hline
\end{tabular}


In Table 5, all variables which are extracted from the model and their data are transferred to fuzzy data. The change from crisp data to fuzzy data is based on Table 4. Based on the FIS logic and following an OECD (Organisation for Economic Co-operation and Development) rating concept based on seven categories or classes, the data is classified, that is, the rankings of customer companies are determined.

The membership function of each class is shown below in Figure 1. The figure presents an overview of the seven individual membership functions which are mathematically specified in Equations (4)-(10). Some researchers believe that the use of the Mamdani and Sugeno methods yields the same results $[64,65]$.

$$
\begin{aligned}
& \mu_{\widetilde{A_{7}}}=\left\{\begin{array}{c}
\frac{x-16.67}{-2.97+16.67},-16.67 \leq x \leq-2.97 \\
\frac{16.67-x}{16.67+2.97},-2.97 \leq x \leq 16.67 \\
0, \quad \text { otherwise }
\end{array}\right. \\
& \mu_{\widetilde{A_{6}}}=\left\{\begin{array}{c}
\frac{x-0}{16.67-0}, 0 \leq x \leq 16.67 \\
\frac{33.33-x}{33.33-16.67}, 16.67 \leq x \leq 33.33 \\
0, \quad \text { otherwise }
\end{array}\right. \\
& \mu_{\widetilde{A_{5}}}=\left\{\begin{array}{c}
\frac{x-16.67}{33.33-16.67}, 16.67 \leq x \leq 33.33 \\
\frac{50-x}{50-33.33}, 33.33 \leq x \leq 50 \\
0, \text { otherwise }
\end{array}\right. \\
& \mu_{\widetilde{A_{4}}}=\left\{\begin{array}{c}
\frac{x-33.33}{50-33.33}, 33.33 \leq x \leq 50 \\
\frac{66.67-x}{66.67-50}, 50 \leq x \leq 66.67 \\
0, \text { otherwise }
\end{array}\right. \\
& \mu_{\widetilde{A_{3}}}=\left\{\begin{array}{c}
\frac{x-50}{66.67-50}, 50 \leq x \leq 66.67 \\
\frac{83.33-x}{83.33-66.67}, 66.67 \leq x \leq 83.33 \\
0, \text { otherwise }
\end{array}\right. \\
& \mu_{\widetilde{A_{2}}}=\left\{\begin{array}{c}
\frac{x-66.67}{83.33-66.67}, 66.67 \leq x \leq 83.33 \\
\frac{10-x}{100-83.33}, 83.33 \leq x \leq 100 \\
0, \text { otherwise }
\end{array}\right. \\
& \mu_{\widetilde{A_{1}}}=\left\{\begin{array}{c}
\frac{x-83.33}{100-83.33}, 83.33 \leq x \leq 100 \\
\frac{116.7-x}{116.7-100}, 100 \leq x \leq 116.7 \\
0, \text { otherwise }
\end{array}\right.
\end{aligned}
$$

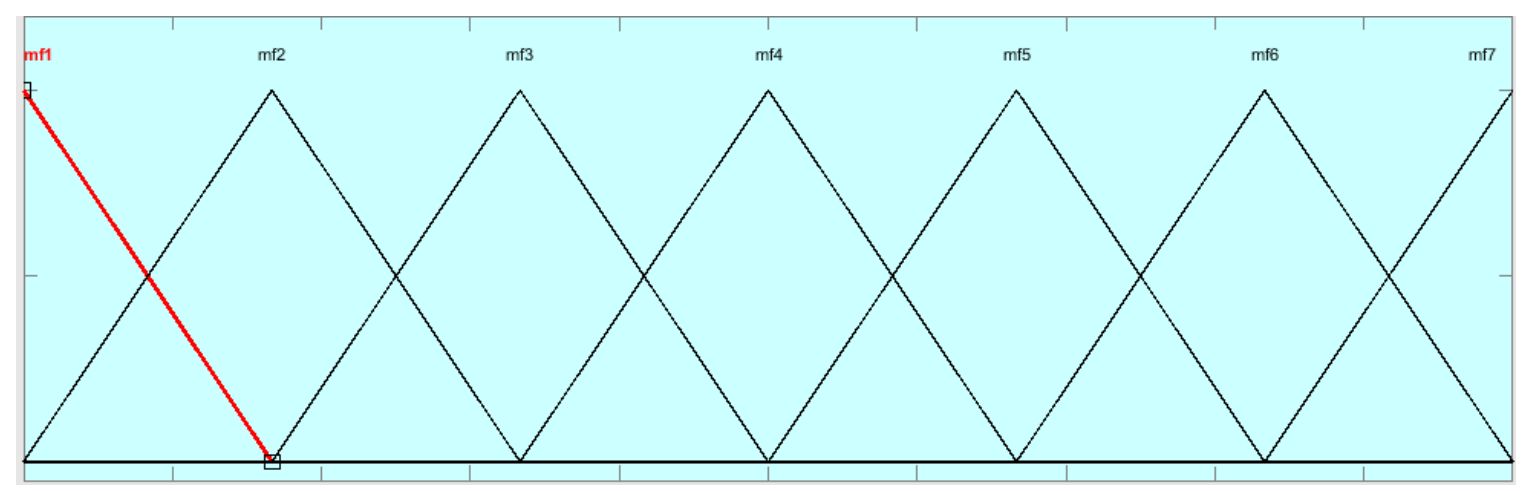

Figure 1. Membership function of output variable. 
With regard to the Export Guarantee Fund of Iran, each company was placed into one of the seven categories. Category 7 attributes to a company the highest risk and probability of default, whereas a category 1 placement represents the lowest risk and the lowest probability of default. Managers can use these membership functions to determine whether they will do business with a company.

\section{Conclusions}

In this uncertain world, most managers attempt to make decisions with the help of managerial tools. Based on these tools, managers can make accurate decisions in areas such as economics, politics, and finance. An important goal is to increase the economic growth rate of countries through exporting. Many nations have established ECAs to support exporters and avoid trade risks. However, because the situation of each country is unique, each agency must create a customized model to help agents analyze credit ratings in their specific countries. In this study, we extracted the key variables for credit rating using the Delphi method. Among the 23 possible variables extracted using Moody's method, only 19 were classified as suitable. We used a fuzzy inference system to determine the credit rating membership function. We then separated the output membership functions into seven categories. Based on these variables and the range of each variable, we used IF-THEN rules to measure the output membership functions to show how these variables affect credit ratings and how credit is allocated to each category based on the membership function.

The proposed method offers the following advantages for determining the credit ratings of companies. First, the proposed FIS method helps managers of the EGFI to rate companies in an uncertain environment. It allows them to determine the risk and the probability of default of a company. Second, the experts of EGFI evaluated the credit rating input variables using the Delphi method. They selected 19 suitable input variables to enter into the FIS method. Third, the FIS method considers not only quantitative ratings but also qualitative values and linguistic terms in an uncertain environment. This method is practical for rating the creditworthiness of companies in the real world.

As mentioned above, the described FIS was customized for the Export Guarantee Fund of Iran (EGFI) for evaluating the credit ratings of buyer companies to determine whether to grant credit to exporters. Due to the general economic situation of Iran and budget limitations, it is crucial to support respective decisions by a well-designed software tool. It will be part of future research to further evaluate the use of the model and its results in the given application scenario.

Apart from the specific FIS developed and applied during our study, the paper shows in general how the considered methodologies can be used in practice. This should help applying the techniques in other settings as well.

For future research, the proposed procedure and FIS model may be applied to other credit rating systems in other countries. In particular, related research may provide further insights regarding a broader empirical validity of obtained information (such as ranges in Table 4 or IF-THEN rules in Table 5).

Author Contributions: A.K.Y. contributed about writing Abstract, literature review and data analysis. T.H. contributed to the discussion of results and provided input for several internal reviews of the article. He also managed the submission processes was responsible for reviews addressing the comments of reviewers and editors. Y.J.W. inspired the subject of paper, handle of paper and provided input for several internal reviews of the article especially help to A.K.Y. for writing this paper. H.-M.W. provided input for several internal reviews of the article especially help to A.K.Y. writing this paper and guide him about finding suitable variables and techniques for solving problems.

Funding: This research received no external funding.

Conflicts of Interest: The authors declare no conflict of interest

\section{References}

1. Zadeh, L.A. Fuzzy sets. Inf. Control 1965, 8, 338-353. [CrossRef] 
2. Pamucar, D.; Ćirović, G. Vehicle route selection with an adaptive neuro fuzzy inference system in uncertainty conditions. Decis. Mak. Appl. Manag. Eng. 2018, 1, 13-37. [CrossRef]

3. Sremac, S.; Tanackov, I.; Kopić, M.; Radović, D. ANFIS model for determining the economic order quantity. Decis. Mak. Appl. Manag. Eng. 2018, 1, 81-92. [CrossRef]

4. Lukovac, V.; Pamučar, D.; Popović, M.; Đorović, B. Portfolio model for analyzing human resources: An approach based on neuro-fuzzy modeling and the simulated annealing algorithm. Expert Syst. Appl. 2017, 90, 318-331. [CrossRef]

5. Pamučar, D.; Ljubojević, S.; Kostadinović, D.; Đorović, B. Cost and risk aggregation in multi-objective route planning for hazardous materials transportation-A neuro-fuzzy and artificial bee colony approach. Expert Syst. Appl. 2016, 65, 1-15. [CrossRef]

6. Pamučar, D.; Vasin, L.; Atanasković, P.; Miličić, M. Planning the City Logistics Terminal Location by Applying the Green-Median Model and Type-2 Neurofuzzy Network. Comput. Intell. Neurosci. 2016, 2016, 6972818. [CrossRef] [PubMed]

7. Akkoç, S. An empirical comparison of conventional techniques, neural networks and the three stage hybrid Adaptive Neuro Fuzzy Inference System (ANFIS) model for credit scoring analysis: The case of Turkish credit card data. Eur. J. Oper. Res. 2012, 222, 168-178. [CrossRef]

8. Ramkumar, M.; Busi, M. A modified ANP and fuzzy inference system based approach for risk assessment of in-house and third party e-procurement systems. Strateg. Outsourcing Int. J. 2016, 9, 159-188. [CrossRef]

9. Yazdi, H.S.; Pourreza, R.; Sadoghi Yazdi, M. Constraint learning using adaptive neural-fuzzy inference system. Int. J. Intell. Comput. Cybern. 2010, 3, 257-278. [CrossRef]

10. Moghadam, S.A.; Karimi, M.; Sadi Mesgari, M. Application of a fuzzy inference system to mapping prospectivity for the Chahfiroozeh copper deposit, Kerman, Iran. J. Spat. Sci. 2015, 60, 233-255. [CrossRef]

11. Dash, R.; Dash, P. Efficient stock price prediction using a Self Evolving Recurrent Neuro-Fuzzy Inference System optimized through a Modified technique. Expert Syst. Appl. 2016, 52, 75-90. [CrossRef]

12. Mihaelajméno, M.O.O. Credit Rating Agency Performance in Terms of Profit. Procedia Econ. Financ. 2015, 30, 631-642. [CrossRef]

13. Gheissari, A. Contemporary Iran: Economy, Society, Politics; Oxford University Press: New York, NY, USA, 2009.

14. Al-Najjar, D.; Al-Najjar, B. Developing a multi stage predicting system for corporate credit rating in emerging markets: Jordanian case. J. Enterp. Inf. Manag. 2014, 27, 475-487. [CrossRef]

15. Bian, J. Regulating the Chinese credit rating agencies: Progress and challenges. J. Money Laund. Control 2015, 18, 66-80. [CrossRef]

16. Chen, Y.-S.; Cheng, C.-H. Hybrid models based on rough set classifiers for setting credit rating decision rules in the global banking industry. Knowl. Based Syst. 2013, 39, 224-239. [CrossRef]

17. Doumpos, M.; Niklis, D.; Zopounidis, C.; Andriosopoulos, K. Combining accounting data and a structural model for predicting credit ratings: Empirical evidence from European listed firms. J. Bank. Financ. 2015, 50, 599-607. [CrossRef]

18. Gibilaro, L.; Mattarocci, G. Pricing policies and customers' portfolio concentration for rating agencies: Evidence from Fitch, Moody's and S\&P. Acad. Bank. Stud. J. 2011, 10, 23-51.

19. Gogas, P.; Papadimitriou, T.; Agrapetidou, A. Forecasting bank credit ratings. J. Risk Financ. 2014, 15, $195-209$. [CrossRef]

20. Orsenigo, C.; Vercellis, C. Linear versus nonlinear dimensionality reduction for banks' credit rating prediction. Knowl. Based Syst. 2013, 47, 14-22. [CrossRef]

21. Ozturk, H.; Namli, E.; Erdal, H.I. Modelling sovereign credit ratings: The accuracy of models in a heterogeneous sample. Econ. Model. 2016, 54, 469-478. [CrossRef]

22. Pasricha, P.; Selvamuthu, D.; Viswanathan, A. Markov regenerative credit rating model. J. Risk Financ. 2017, 18, 311-325. [CrossRef]

23. $\mathrm{Hu}, \mathrm{H}$.; $\mathrm{Hu}, \mathrm{H}$. The impact of sovereign rating events on bank stock returns: An empirical analysis for the Eurozone. J. Risk Financ. 2017, 18, 338-367. [CrossRef]

24. Mamdani, E.H.; Assilian, S. An experiment in linguistic synthesis with a fuzzy logic controller. Int. J. Man-Mach. Stud. 1975, 7, 1-13. [CrossRef]

25. Mamdani, E.H. Application of fuzzy algorithms for control of simple dynamic plant. Proc. Inst. Electr. Eng. 1974, 121, 1585-1588. [CrossRef] 
26. Sugeno, M. Theory of Fuzzy Integrals and Its Applications. Ph.D. Thesis, Tokyo Institute of Technology, Tokyo, Japan, 1974.

27. Takagi, T.; Sugeno, M. Fuzzy identification of systems and its applications to modeling and control. IEEE Trans. Syst. Man Cybern. 1985, 15, 116-132. [CrossRef]

28. Anderson, R.C.; Mansi, S.A.; Reeb, D.M. Founding family ownership and the agency cost of debt. J. Financ. Econ. 2003, 68, 263-285. [CrossRef]

29. Syau, Y.-R.; Hsieh, H.-T.; Lee, E.S. Fuzzy numbers in the credit rating of enterprise financial condition. Rev. Quant. Financ. Account. 2001, 17, 351-360. [CrossRef]

30. Asquith, P.; Beatty, A.; Weber, J. Performance pricing in bank debt contracts. J. Account. Econ. 2005, 40, 101-128. [CrossRef]

31. Baghai, R.P.; Servaes, H.; Tamayo, A. Have rating agencies become more conservative? Implications for capital structure and debt pricing. J. Financ. 2014, 69, 1961-2005. [CrossRef]

32. An, X.; Deng, Y.; Sanders, A.B. Subordination level as a predictor of credit risk. In Proceedings of the Cambridge-UNC Charlotte Symposium, Cambridge, UK, 12-14 June 2006.

33. Kong, D.; Tiong, R.L.; Cheah, C.Y.; Permana, A.; Ehrlich, M. Assessment of credit risk in project finance. J. Constr. Eng. Manag. 2008, 134, 876-884. [CrossRef]

34. Gray, S.; Mirkovic, A.; Ragunathan, V. The determinants of credit ratings: Australian evidence. Aust. J. Manag. 2006, 31, 333-354. [CrossRef]

35. Min, J.H.; Lee, Y.-C. A practical approach to credit scoring. Expert Syst. Appl. 2008, 35, 1762-1770. [CrossRef]

36. Huang, Z.; Chen, H.; Hsu, C.-J.; Chen, W.-H.; Wu, S. Credit rating analysis with support vector machines and neural networks: A market comparative study. Decis. Support Syst. 2004, 37, 543-558. [CrossRef]

37. Barry, P.J.; Escalante, C.L.; Ellinger, P.N. Credit risk migration analysis of farm businesses. Agric. Financ. Rev. 2002, 62, 1-11. [CrossRef]

38. Dincer, H.; Yuksel, S.; Hacioglu, U. CAMELS-based Determinants for the Credit Rating of Turkish Deposit Banks. Int. J. Financ. Bank. Stud. 2015, 4, 1.

39. Hajek, P.; Michalak, K. Feature selection in corporate credit rating prediction. Knowl. Based Syst. 2013, 51, 72-84. [CrossRef]

40. Hossain, S.B. Credit Rating Methodology of the Bangladesh Rating Agency Limited; UIU Institutional Reposity: Dhaka, Bangladesh, 2018.

41. Yang, C.-C.; Ou, S.-L.; Hsu, L.-C. A Hybrid Multi-Criteria Decision-Making Model for Evaluating Companies' Green Credit Rating. Sustainability 2019, 11, 1506. [CrossRef]

42. Gül, S.; Kabak, Ö.; Topcu, Y.İ. An OWA Operator-Based Cumulative Belief Degrees Approach for Credit Rating. Int. J. Intell. Syst. 2018, 33, 998-1026. [CrossRef]

43. Mishra, S.; Bansal, R. Credit rating and its interaction with financial ratios: A study of BSE 500 companies. In Behavioral Finance and Decision-Making Models; IGI Global: Hershey, PA, USA, 2019; pp. 251-268.

44. Chi, G.; Zhang, Y.; Shi, B. The debt rating for small enterprises based on Probit regression. J. Manag. Sci. China 2016, 19, 136-156.

45. Gül, S.; Kabak, Ö.; Topcu, I. A multiple criteria credit rating approach utilizing social media data. Data Knowl. Eng. 2018, 116, 80-99. [CrossRef]

46. Du, Y. Enterprise Credit Rating Based on Genetic Neural Network. In MATEC Web of Conferences; EDP Sciences: Les Ulis, France, 2018; Volume 227, p. 2011.

47. Kashif, M.; Ahmed, J.; Islam, M.; Gillani, U.F. Effect of Credit Rating on Trade Credit: Empirical Evidences from Pakistani Non-financial Firms. Int. J. Econ. Financ. Res. Appl. 2019, 3, 1-10.

48. Li, Z.; Zheng, X. A Critical Study of Commercial Banks' Credit Risk Assessment and Management for SMEs: The Case of Agricultural Bank of China. J. Appl. Manag. Invest. 2017, 6, 106-117.

49. Akdoğu, E.; Alp, A. Credit risk and governance: Evidence from credit default swap spreads. Financ. Res. Lett. 2016, 17, 211-217. [CrossRef]

50. Grassa, R. Corporate governance and credit rating in Islamic banks: Does Shariah governance matters? J. Manag. Gov. 2016, 20, 875-906. [CrossRef]

51. Kachwala, S.T.; Singla, C. Impact of Ownership Structure on Agency Cost of Debt in India; Indian Institute of Management Ahmedabad: Ahmedabad, India, 2016.

52. Chodnicka-Jaworska, P. Macroeconomic aspects of banks' credit ratings. Equilib. Q. J Econ. Econ. Policy 2017, 12, 101-120. [CrossRef] 
53. D'Apice, V.; Ferri, G.; Lacitignola, P. Rating Performance and Bank Business Models: Is There a Change with the 2007-2009 Crisis? Ital. Econ. J. 2016, 2, 385-420. [CrossRef]

54. Sariannidis, N.; Papadakis, S.; Garefalakis, A.; Lemonakis, C.; Kyriaki-Argyro, T. Default avoidance on credit card portfolios using accounting, demographical and exploratory factors: Decision making based on machine learning (ML) techniques. Ann. Oper. Res. 2019, 277, 1-25. [CrossRef]

55. Cuny, C.; Dube, S. Does reporting quality moderate the relation between economic changes and changes in the cost of debt? In Proceedings of the 28th Annual Conference on Financial Economics and Accounting, Philadelphia, PA, USA, 10-11 November 2017.

56. DeBoskey, D.; Li, Y.; Lobo, G.J.; Luo, Y. Transparency of Corporate Political Disclosure and the Cost of Debt; Working Paper; San Diego State University: San Diego, CA, USA, 2017.

57. Boumparis, P.; Milas, C.; Panagiotidis, T. Fair or not? How credit rating agencies calculated their ratings during the Eurozone crisis. Available online: http://eprints.lse.ac.uk/70340/ (accessed on 15 April 2019).

58. Chang, J.-H.; Hung, M.-W.; Tsai, F.-T. Credit contagion and competitive effects of bond rating downgrades along the supply chain. Financ. Res. Lett. 2015, 15, 232-238. [CrossRef]

59. Saleh, M.H.; Jaber, J.J.; Al-khawaldeh, A.A. The role of credit scoring, cost and product discrimination in improving the competitiveness of Jordanian insurance companies. Int. J. Econ. Financ. 2016, 8, 252-259. [CrossRef]

60. Saha, N.; Paul, K.C. Impact of Credit Rating on the Corporate Rate of Interest and Investment Flow: A Study with Reference to Some Selected Indian Companies. Ph.D. Thesis, Vidyasagar University, Midnapore, India, 2017.

61. Pap, M.; Homolya, D. Measurement and mitigation of country risk: The role of quantitative and qualitative factors, Insurance market trends. In Proceedings of the 8th International Scientific Conference "Future World by 2050", Pula, Croatia, 1-3 June 2017; Volume 1, p. 193.

62. Sahi, C.A.I.; Rasheed, A. Impact of sovereign credit rating and country risk on bond market of Pakistan. Paradigms 2017, 11, 1h.

63. Bojadziev, G.; Bojadziev, M. Fuzzy Sets, Fuzzy Logic, Applications; World Scientific: Singapore, 1995 ; Volume 5.

64. Kaur, A.; Kaur, A. Comparison of fuzzy logic and neuro-fuzzy algorithms for air conditioning system. Int. J. Soft Comput. Eng. 2012, 2, 417-420.

65. Shleeg, A.A.; Ellabib, I.M. Comparison of mamdani and sugeno fuzzy interference systems for the breast cancer risk. Int. J. Comput. Inf. Sci. Eng. 2013, 7, 387-391. 